\title{
Activity of lipophilic and hydrophilic drugs against dormant and replicating Mycobacterium tuberculosis
}

\author{
Giovanni Piccaro ${ }^{1}$, Giovanna Poce ${ }^{2}$, Mariangela Biava ${ }^{2}$, Federico Giannoni ${ }^{1}$ and Lanfranco Fattorini ${ }^{1}$
}

The Journal of Antibiotics (2015) 68, 711-714; doi:10.1038/ja.2015.52; published online 6 May 2015

\begin{abstract}
Mycobacterium tuberculosis is responsible for about 9 million new tuberculosis (TB) cases per year and 2 billion latent TB infections (LTBI) worldwide. ${ }^{1,2}$ Most of active TB is owing to the reactivation of LTBI in patients in which M. tuberculosis lies dormant in their tissues. Current treatments require 6 months of combination therapy with isoniazid, rifampin, pyrazinamide and ethambutol for active $\mathrm{TB}$, and 9 months of isoniazid or 3 months of rifapentine plus isoniazid for LTBI. LTBI and active TB comprise heterogeneous mixtures of cellular and caseous granulomas containing $M$. tuberculosis bacilli ranging from replicating to dormant, nonreplicating, stages. In cellular granulomas, replicating bacilli are killed by current therapy. In contrast, in low-vascularized caseous granulomas low oxygen pressure restricts the growth of replicating to microaerophilic/anaerobic M. tuberculosis in their hypoxic, necrotic centers, allowing bacilli to transit into a dormant state. In these lesions, without the assistance of blood supply, drugs do not diffuse into caseum, a material containing several lipids including cholesterol, triacylglycerols and lactosylceramide. ${ }^{3}$ Current therapies eliminate replicating but not nonreplicating $M$. tuberculosis, thus new regimens are needed to shorten anti-TB treatments.
\end{abstract}

To mimic the hypoxic and acidic environment of TB granulomas, we previously measured the activity of anti-TB drugs and nitrocompounds against aerobic (replicating), and hypoxic (slowly replicating and nonreplicating) $M$. tuberculosis by the Wayne model at $\mathrm{pH} 5.8$ set up in our laboratory. ${ }^{4,5}$ Using the same model, we investigated here the tuberculocidal effect of repurposed drugs (clofazimine, linezolid, meropenem \pm clavulanic acid, thioridazine), last approved anti-TB drugs (rifapentine, bedaquiline) ${ }^{1,2}$ and a new antimycobacterial agent, the BM212 analogue (a 1,5-diphenyl pyrrole) BM635 $^{6}$ (Supplementary Figure S1) against M. tuberculosis strain H37Rv. Briefly, mycobacteria were grown in 20- by $125-\mathrm{mm}$ screwcap tubes containing dubos-tween-albumin (DTA) broth acidified at $\mathrm{pH} 5.8$ and stirred with 8-mm magnetic bars. ${ }^{5}$ For the preparation of aerobic cells, logarithmically growing cultures were diluted in DTA broth to about $1 \times 10^{6} \mathrm{CFU} \mathrm{ml}^{-1}$ and transferred to tubes in $12-\mathrm{ml}$ volumes. Tubes were incubated at $37^{\circ} \mathrm{C}$ with loosened screw caps and fast stirring. For the preparation of hypoxic cells, logarithmically growing cultures were diluted in DTA broth to about $1 \times 10^{6} \mathrm{CFU} \mathrm{ml}{ }^{-1}$ and transferred to tubes in 16-ml volumes but in this case, to obtain anaerobic conditions, the caps were tightly screwed and tight rubber caps were put under the caps. Tubes were incubated with slow stirring (120 r.p.m.) at $37^{\circ} \mathrm{C}$. To determine drug activity, 5-day-old aerobic cultures (A5, replicating), and 5-, 12- and 19-day-old hypoxic cultures (H5, slowly replicating; $\mathrm{H} 12$ and H19, nonreplicating) were incubated with drugs for 7, 14 and 21 days. ${ }^{5}$ Drugs were added to A5 cultures by micropipette and to H5, H12 and H19 cultures by a syringe. Clofazimine, linezolid, meropenem, thioridazine, rifapentine and bedaquiline were tested at their maximum drug concentration in serum $\left(C_{\max }\right)$ at $1,8,20,0.5,10$ and $1 \mu \mathrm{g} \mathrm{ml}^{-1}$, respectively. Clavulanic acid was tested at $2.5 \mu \mathrm{g} \mathrm{ml}^{-1}$. BM635 was tested at $11 \mu \mathrm{g} \mathrm{ml}^{-1}$, corresponding to the MIC in the low oxygen recovery assay. ${ }^{6}$ After incubation, $1 \mathrm{ml}$ of aerobic or hypoxic culture was washed and resuspended in $1 \mathrm{ml}$ of DTA broth, and $0.2 \mathrm{ml}$ was inoculated in Middlebrook $7 \mathrm{H} 10$ agar plates, which were incubated at $37^{\circ} \mathrm{C}$ under $5 \% \mathrm{CO}_{2}$ for 3 weeks for CFU determination.

Drug activities against A5, H5, H12 and H19 bacilli are shown in Figure 1. Among the repurposed drugs (Figure 1a-d), clofazimine was the most active one against all four cell stages. Linezolid was effective against $\mathrm{A} 5$ and $\mathrm{H} 5$ but not $\mathrm{H} 12$ and $\mathrm{H} 19$ cells. Meropenem was less potent than linezolid against $\mathrm{A} 5$ and $\mathrm{H} 5$ cells $\left(2.9\right.$ and $0.5-\log _{10}$-CFU reduction on day 21, respectively) and almost inactive against $\mathrm{H} 12$ and $\mathrm{H} 19$ cells. Addition of clavulanic acid to meropenem increased killing of A5 and $\mathrm{H} 5$ cells by at most $1.2-\log _{10}$ CFU. Thioridazine was ineffective against A5, H5, H12, but active against $\mathrm{H} 19$ cells (1.4- $\log _{10}$-CFU reduction on day 21). It is important to point out that after 14 days of treatment of A5 cells with linezolid, meropenem and meropenem+clavulanic acid, $M$. tuberculosis re-grew on day 21 , likely owing to development of resistant mutants which are known to be generated at the concentrations used here for linezolid and meropenem. ${ }^{7,8}$

As to last approved and new drugs (Figure 1e-h), rifapentine was the most effective one, with CFU reductions on day 21 of $7.9-\log _{10}$ against $\mathrm{A} 5$ cells and $\geqslant 5.1-\log _{10}$ against $\mathrm{H} 5, \mathrm{H} 12$ and $\mathrm{H} 19$ cells. Bedaquiline showed high activity against $\mathrm{A} 5$ cells $\left(5.8-\log _{10}\right.$-CFU reduction on day 21 ) and $\mathrm{H} 5, \mathrm{H} 12$ and $\mathrm{H} 19$ cells (about $3-\log _{10^{-}}$ CFU-reduction on day 21). The BM635 compound showed a behavior

${ }^{1}$ Department of Infectious, Parasitic and Immune-Mediated Diseases, Istituto Superiore di Sanità, Rome, Italy and ${ }^{2}$ Department of Drug Chemistry and Technologies, Sapienza University of Rome, Rome, Italy

Correspondence: Dr L Fattorini, Department of Infectious, Parasitic and Immune-Mediated Diseases, Istituto Superiore di Sanità, Viale Regina Elena 299, Rome 00161, Italy. E-mail: lanfranco.fattorini@iss.it

Received 5 February 2015; revised 12 March 2015; accepted 8 April 2015; published online 6 May 2015 
a

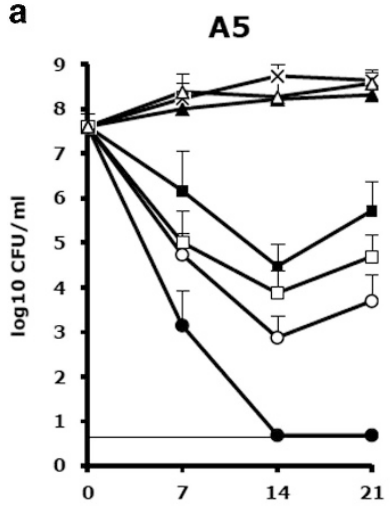

e

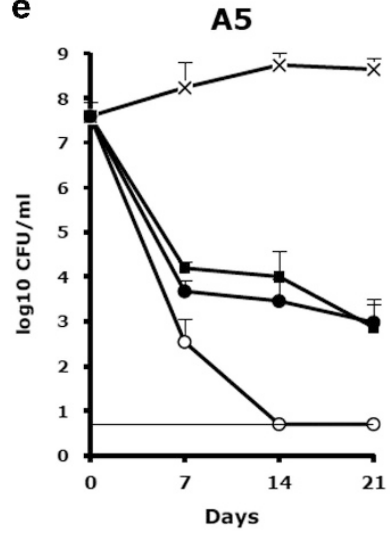

b

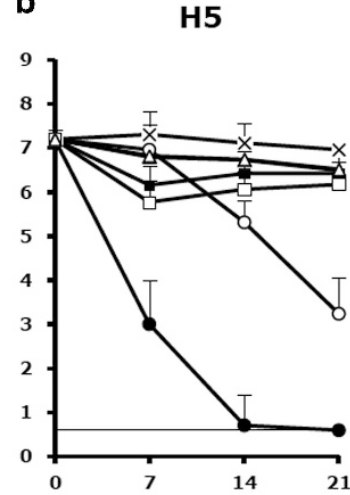

f

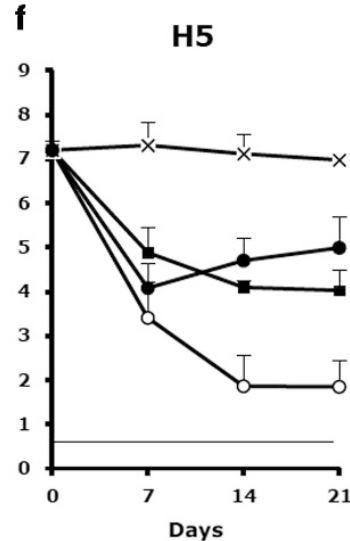

c

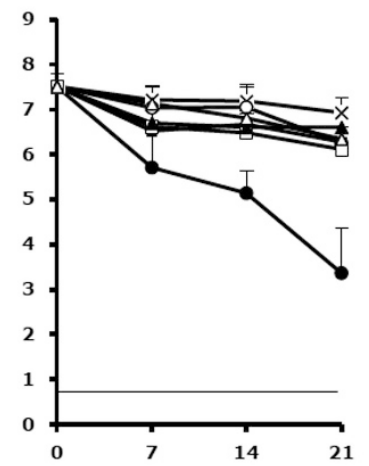

H12

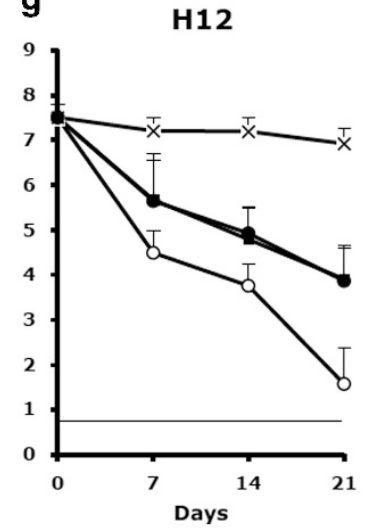

d

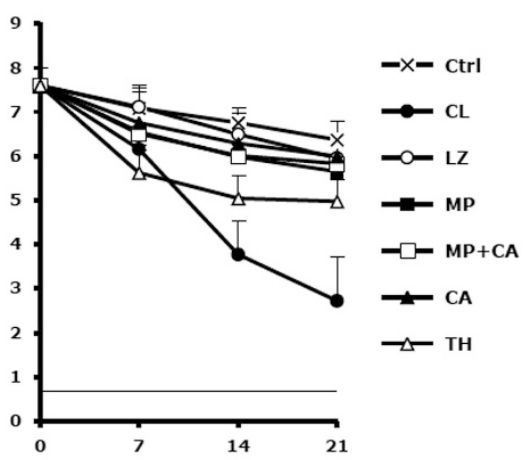

h $\quad H 19$

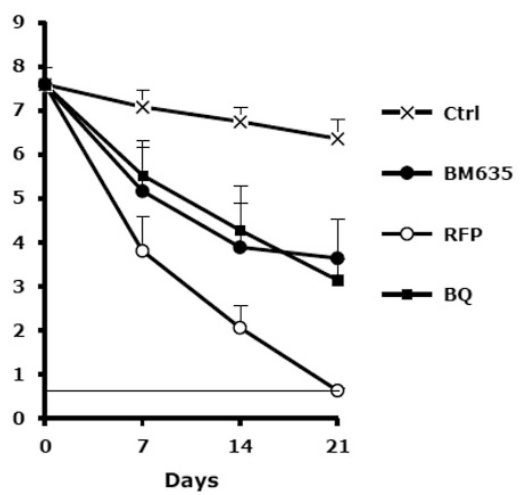

Figure 1 Activity of drugs against aerobic and hypoxic M. tuberculosis. CFU of $M$. tuberculosis grown in aerobic and hypoxic acidic conditions after 0 , 7,14 and 21 days of drug exposure are shown. Five-day-old aerobic (A5) cultures, and 5-, 12- and 19-day-old hypoxic (H5, H12 and H19, respectively) cultures were incubated with drugs. Ctrl, control; CL, clofazimine; LZ, linezolid; MP, meropenem; MP+CA, meropenem plus clavulanic acid; CA, clavulanic acid; TH, thioridazine; BM635; RFP, rifapentine; BQ, bedaquiline. The drug concentrations used were: 1, 8, 20, 20+2.5, 2.5, 0.5, 11, 10, $1 \mu \mathrm{g}$ ml-1, respectively. Dashed lines indicate the limit of detection ( $5 \mathrm{CFU} \mathrm{ml} \mathrm{ml}^{-1}$ ). Mean and standard deviations from two experiments are shown.

similar to that of bedaquiline against A5, H12 and H19, but not H5 bacilli, which re-grew after day 7. Overall, rifapentine, bedaquiline, clofazimine and BM635 were the most active drugs, with $\geqslant 2-\log _{10}$-CFU reductions in all the four cell stages in $\leqslant 14$ days. In previous studies, performed by using the same aerobic and hypoxic acidic conditions used above, we tested the activity of 10 other drugs at their $C_{\max }$ and showed that also rifampin, PA-824 and nitazoxanide decreased A5, H5, H12 and H19 CFU by $\geqslant 2-\log _{10} .{ }^{5}$

Recent studies indicated that compounds with anti-TB activity are more lipophilic than the inactive ones. ${ }^{9-12}$ Lipophilicity is mostly expressed as $\log \mathrm{P}$, i.e., the logarithm of the partition coefficient in a specific solvent $\left(\mathrm{P}_{\text {octanol }} / \mathrm{P}_{\text {water }}\right){ }^{9}{ }^{9}$ Drugs with $\log P<0$ are hydrophilic. ${ }^{13}$ According to the Lipinski rule-of-five, compounds with moderate lipophilicity ( $\log \mathrm{P}$ between 0 and 3 ) are optimal for oral administration owing to a good balance of solubility and permeability. ${ }^{13}$ Ninety percent of marketed drugs have a $\log \mathrm{P}$ value in the range of $0-5$. Instead, highly lipophilic compounds $(\log \mathrm{P}>5)$ tend to accumulate in the lipoidal areas of tissues. Many synthetically derived TB agents are outside the drug-chemical space of the Lipinski rule-of-five. ${ }^{11}$ Indeed, some of the recently developed anti-TB drugs have high lipophilicity. In the drug pipeline reported at http://www. newtbdrugs.org/pipeline.php, the $\log \mathrm{P}$ predicted values calculated by ALOGPS at http://www.drugbank.ca or reported in the literature ${ }^{11}$ for phase III drugs are: bedaquiline, 6.37; delamanid, 6.14; rifapentine, 4.83; moxifloxacin, 0.01; gatifloxacin, -0.23 , and for phase II drugs are: PA-824, 2.8; SQ-109, 4.64; sutezolid, 1.22; AZD-5847, 0.78; linezolid, 0.61. Current 6-month therapy includes 1 lipophilic drug (rifampin, $\log \mathrm{P} 3.85$ ) and 3 hydrophilic agents (isoniazid, ethambutol and pyrazinamide: $\log \mathrm{P}-0.71,-0.12$ and -0.71 , respectively). In recently reported clinical phase III trials, current 6-month therapy was not improved after replacement of isoniazid and/or ethambutol with moxifloxacin and gatifloxacin. ${ }^{14,15}$ However, a 6-month regimen in which isoniazid was replaced by daily moxifloxacin for 2 months followed by one weekly dose of rifapentine and moxifloxacin for 4 months was as effective as the current regimen. ${ }^{16}$ Several other clinical trials containing lipophilic compounds such as bedaquiline, delamanid, PA-824, rifapentine, SQ-109, sutezolid, AZD-5847, linezolid and clofazimine $(\log \mathrm{P} 7.81)$ are in progress. ${ }^{17}$ As to LTBI treatment, 3 months of rifapentine plus isoniazid is as effective as 9 months of isoniazid alone. ${ }^{1}$ Overall, this information corroborates the general idea that lipophilicity of a compound reflects partitioning into the hydrophobic phase of M. tuberculosis cell wall and, possibly, of caseum, and suggests that lipophilic drugs may be important for antiTB treatment.

Some studies showed that, within each drug class, lipophilic derivatives were more active against mycobacteria than their hydrophilic companions. ${ }^{12}$ However, this trend was reported for replicating mycobacteria and not for nonreplicating M. tuberculosis. Here, to fill in the gap of information, we compared $\geqslant 2-\log _{10}$-CFU reductions of A5 (replicating, aerobes), H5 (slowly replicating, microaerophiles) and H12 and H19 (nonreplicating, anaerobes) cells of this study and of our previous study ${ }^{5}$ (18 single drugs in total) with lipophilicities of the 
Growth stage and days of exposure

\begin{tabular}{|c|c|c|c|c|c|c|c|c|c|c|c|c|c|c|}
\hline \multirow[b]{2}{*}{ Drug } & \multicolumn{3}{|c|}{ A5 } & \multicolumn{3}{|c|}{ H5 } & \multicolumn{3}{|c|}{ H12 } & \multicolumn{3}{|c|}{ H19 } & \multirow[b]{2}{*}{ Reference } & \multirow[b]{2}{*}{$\log \mathrm{P}^{\mathrm{a}}$} \\
\hline & 7 & 14 & 21 & 7 & 14 & 21 & 7 & 14 & 21 & 7 & 14 & 21 & & \\
\hline Rifampin & $\bullet$ & $\bullet$ & $\bullet$ & $\bullet$ & $\bullet$ & $\bullet$ & $\bullet$ & $\bullet$ & $\bullet$ & $\bullet$ & $\bullet$ & $\bullet$ & 5 & 3.85 \\
\hline Rifapentine & $\bullet$ & $\bullet$ & $\bullet$ & $\bullet$ & $\bullet$ & $\bullet$ & $\bullet$ & $\bullet$ & $\bullet$ & $\bullet$ & $\bullet$ & $\bullet$ & this study & 4.83 \\
\hline Bedaquiline & $\bullet$ & $\bullet$ & $\bullet$ & $\bullet$ & $\bullet$ & $\bullet$ & $\circ$ & $\bullet$ & $\bullet$ & $\circ$ & $\bullet$ & $\bullet$ & this study & 6.37 \\
\hline Clofazimine & $\bullet$ & $\bullet$ & $\bullet$ & $\bullet$ & $\bullet$ & $\bullet$ & $\circ$ & $\bullet$ & $\bullet$ & $\circ$ & $\bullet$ & $\bullet$ & this study & 7.39 \\
\hline Nitazoxanide & $\bullet$ & $\bullet$ & $\bullet$ & $\circ$ & $\bullet$ & $\bullet$ & $\circ$ & $\circ$ & $\bullet$ & $\circ$ & $\bullet$ & $\bullet$ & 5 & 2.14 \\
\hline PA-824 & $\bullet$ & $\bullet$ & $\bullet$ & $\bullet$ & 0 & $\circ$ & $\circ$ & $\circ$ & $\bullet$ & $\circ$ & $\bullet$ & $\bullet$ & 5 & 2.8 \\
\hline BM635 & $\bullet$ & $\bullet$ & $\bullet$ & $\bullet$ & $\circ$ & $\circ$ & $\circ$ & $\bullet$ & $\bullet$ & $\circ$ & $\bullet$ & $\bullet$ & this study & $4.57^{6}$ \\
\hline Metronidazole & 0 & $\circ$ & $\circ$ & 0 & $\circ$ & $\circ$ & 0 & $\circ$ & $\bullet$ & $\circ$ & $\bullet$ & $\bullet$ & 5 & -0.15 \\
\hline Amikacin & $\bullet$ & $\bullet$ & $\bullet$ & $\bullet$ & $\bullet$ & $\bullet$ & $\circ$ & $\circ$ & $\circ$ & $\circ$ & $\circ$ & $\circ$ & 5 & -3.2 \\
\hline Moxifloxacin & $\bullet$ & $\bullet$ & $\bullet$ & $\bullet$ & $\bullet$ & $\bullet$ & $\circ$ & $\circ$ & $\circ$ & $\circ$ & $\circ$ & $\circ$ & 5 & 0.01 \\
\hline Linezolid & $\bullet$ & $\bullet$ & $\bullet$ & $\circ$ & $\circ$ & $\bullet$ & $\circ$ & $\circ$ & $\circ$ & $\circ$ & $\circ$ & $\circ$ & this study & 0.61 \\
\hline Meropenem & $\bullet$ & $\bullet$ & $\bullet$ & 0 & $\circ$ & $\circ$ & $\circ$ & $\circ$ & $\circ$ & $\circ$ & $\circ$ & $\circ$ & this study & -0.69 \\
\hline Meropenem + clavulanic acid & $\bullet$ & $\bullet$ & $\bullet$ & $\circ$ & $\circ$ & $\circ$ & $\circ$ & $\circ$ & $\circ$ & $\circ$ & $\circ$ & $\circ$ & this study & $-0.69,-1.2$ \\
\hline Isoniazid & $\bullet$ & $\bullet$ & 0 & 0 & 0 & $\circ$ & 0 & $\circ$ & 0 & $\circ$ & $\circ$ & $\circ$ & 5 & -0.71 \\
\hline Pyrazinamide & 0 & $\circ$ & $\circ$ & $\circ$ & $\circ$ & $\circ$ & $\circ$ & $\circ$ & $\circ$ & $\circ$ & $\circ$ & $\circ$ & 5 & -0.71 \\
\hline Ethambutol & $\circ$ & $\circ$ & $\circ$ & 0 & $\circ$ & $\circ$ & $\circ$ & $\circ$ & $\circ$ & $\circ$ & $\circ$ & $\circ$ & 5 & -0.12 \\
\hline Clavulanic acid & $\circ$ & $\circ$ & $\circ$ & $\circ$ & $\circ$ & $\circ$ & $\circ$ & $\circ$ & $\circ$ & $\circ$ & $\circ$ & $\circ$ & this study & -1.2 \\
\hline Niclosamide & 0 & 0 & 0 & 0 & 0 & 0 & 0 & $\circ$ & 0 & 0 & $\circ$ & 0 & 5 & 4.49 \\
\hline Thioridazine & $\circ$ & 0 & $\circ$ & $\circ$ & $\circ$ & $\circ$ & $\circ$ & $\circ$ & $\circ$ & $\circ$ & $\circ$ & $\circ$ & this study & 5.93 \\
\hline
\end{tabular}

Figure 2 Activity and lipophilicity (logP) of 18 single drugs and meropenem plus clavulanic acid against $M$. tuberculosis. Activities under aerobic and hypoxic acidic conditions shown in Figure 1 of this study and in our previous study ${ }^{5}$ were pooled, outlined as $\geqslant 2-\log _{10}-\mathrm{CFU} \mathrm{ml}^{-1}$ decreases, and compared with $\log \mathrm{P}$ values. LogP is defined as the $\log \left(\mathrm{P}_{\text {octanol }} / \mathrm{P}_{\text {water }}\right)$, where $\mathrm{P}$ is the partition coefficient for a given compound in a specific solvent. ${ }^{9}$ Symbols: $\mathbf{0}$, $\geqslant 2-\log _{10}-\mathrm{CFU} \mathrm{ml}^{-1}$ decrease; $\circ,<2-\log _{10}$-CFU ml ${ }^{-1}$ decrease. ${ }^{a} \operatorname{logP}$ predicted values were calculated by ALOGPS (http://www.drugbank.ca). ${ }^{b} \log P$ predicted values were calculated by CHILogD. ${ }^{6}$

tested drugs (Figure 2). Overall, we noted that 7 drugs reducing CFU of both nonreplicating $\mathrm{H} 12 / \mathrm{H} 19$ cells and A5/H5 cells by $\geqslant 2-\log _{10}$ (rifampin, rifapentine, bedaquiline, clofazimine, nitazoxanide, PA-824 and BM635) showed a lipophilic character $(\log \mathrm{P} \geqslant 2.14)$. Among the other 11 single compounds, 8 were hydrophilic $(\log \mathrm{P} \leqslant 0.01$ : metronidazole, amikacin, moxifloxacin, meropenem, isoniazid, pyrazinamide, ethambutol and clavulanic acid), 2 were lipophilic $(\log \mathrm{P} \geqslant 4.49$ : niclosamide and thioridazine) and 1 was moderately hydrophilic ( $\log \mathrm{P}$ 0.61: linezolid) but none, with exception of metronidazole, reduced $\mathrm{H} 12$ and $\mathrm{H} 19$ CFU by $\geqslant 2-\log _{10}$. Niclosamide and thioridazine decreased CFU of all the cells by $<2-\log _{10}$. As to current therapy (rifampin+isoniazid+ethambutol+pyrazinamide), only rifampin decreased CFU of all cells by $\geqslant 2 \log _{10}$ whereas isoniazid efficiently killed only A5 cells. In our previous study ${ }^{5}$ the best combination rifampin+PA-824+moxifloxacin+amikacin (2 lipophilic and 2 hydrophilic drugs) sterilized A5, H5, H12 and H19 cells in 14 days (as shown by the lack of regrowth in the MGIT 960 tubes after 100 days of incubation), whereas rifampin+isoniazid+ethambutol +pyrazinamide sterilized A5, H12 and H19 cells in 21 days and failed to kill the $\mathrm{H} 5$ cells. Overall, our observations have to be combined with recent in vivo studies showing that combinations containing the lipophilic compounds bedaquiline, rifapentine, clofazimine, PA-824, sutezolid and the new nitroimidazole TBA-354 ( $\log$ P 4.10) sterilized M. tuberculosis-infected mice in 6-8 weeks whereas rifampin+isoniazid +pyrazinamide did it in 6 months. ${ }^{18,19}$ The observation that in vivo effective $\mathrm{BM} 635^{6}$ is active against replicating, slowly replicating and nonreplicating cells adds to anti-TB armamentarium a novel agent to be tested in combination.

Overall, in the set of compounds tested here, only lipophilic agents killed both dormant H12/H19 cells and A5/H5 cells. Decreased drug permeability may contribute to phenotypic drug resistance of dormant M. tuberculosis, and cell wall alterations in nonreplicating cells were suggested to account for the loss of bactericidal activity of small molecules against them. ${ }^{20}$ It is known that dormant M. tuberculosis remodels cell wall by accumulating free mycolates and lipoarabinomannan, and exporting them into the extracellular matrix. ${ }^{21}$ In its nonreplicating state, intracytoplasmic accumulation of lipid droplets containing trialcylglycerol synthesized from fatty acids derived from host lipids was also observed. ${ }^{22}$ Overall, it could be that increase in the lipid content of dormant cells may be involved in their killing by lipophilic compounds with different mechanism of action studied here. Despite structural changes of nonreplicating cells remain to be more finely elucidated, we suggest that lipophilic agents need to be considered in designing new combinations to be tested in vivo, preceded by pharmacokinetics/pharmakodinamics and toxicity assessments. Besides activity against both nonreplicating and replicating bacilli, lipophilic compounds would also, presumably, have increased availability in areas of lipid-rich caseous necrosis of the lungs of TB patients where nonreplicating M. tuberculosis is believed to reside. Nonreplicating bacilli are particularly important because they constitute the reservoir from which replicating cells emerge when necrotic material of solid caseous granulomas liquefy and discharges through eroded airways with formation of empty cavities. At the luminal surface of these cavities, bacilli are either intracellular (replicating, inside macrophages) or extracellular (nonreplicating/slowly replicating, dispersed within the acellular caseum). Thus, the granulomas are structures which can contain $M$. tuberculosis but that also make chemotherapeutic eradication very difficult owing to the sequestration of dormant bacilli within the caseous centers of necrotic lesions and cavities, where vascular architecture has been destroyed. ${ }^{23,24}$ The lack 
of blood supply to these areas highlights the importance of investigating drug penetration at these sites. MALDI MS imaging is an emerging modality for the visualization of unlabeled drugs in tissue sections of M. tuberculosis-infected lungs. ${ }^{24}$

A recent analysis of human pharmacokinetic parameters revealed positive correlation between $\log \mathrm{P}$ of anti-TB agents and volume of distribution (an indicator of drug distribution into tissues), clearance of the unbound fraction, plasma protein binding. ${ }^{25}$ Thus, drug lipophilicity can influence in vitro and in vivo pharmacokinetics properties.

As such, lipophilic agents may give an important contribution to combination anti-TB therapy in the future. Some support to this view comes from the knowledge that combinations of recently developed, mostly lipophilic, anti-TB drugs sterilized M. tuberculosisinfected mice ${ }^{18,19}$ and are presently tested in promising phase II and III clinical trials. ${ }^{17}$

\section{ACKNOWLEDGEMENTS}

This work was supported in part by the European project StopLATENT-TB grant agreement 200999. We acknowledge Janssen Pharmaceuticals and Sanofi Aventis for the provision of bedaquiline and rifapentine, respectively.

1 Zumla, A. I. et al. New antituberculosis drugs, regimens, and adjunct therapies: needs advances, and future prospects. Lancet Infect. Dis. 14, 327-340 (2014).

2 Poce, G., Cocozza, M., Consalvi, S. \& Biava, M. SAR analysis of new anti-TB drugs currently in pre-clinical and clinical development. Eur. J. Med. Chem. 86, 335-351 (2014).

$3 \mathrm{Kim}, \mathrm{M}$. J. et al. Caseation of human tuberculosis granulomas correlates with elevated host lipid metabolism. EMBO Mol. Med. 2, 258-274 (2010).

4 Wayne, L. G. \& Hayes, L. G. An in vitro model for sequential study of shiftdown of Mycobacterium tuberculosis through two stages of nonreplicating persistence. Infect. Immun. 64, 2062-2069 (1996).

5 Piccaro, G., Giannoni, F., Filippini, P., Mustazzolu, A. \& Fattorini, L. Activities of drug combinations against Mycobacterium tuberculosis grown in aerobic and hypoxic acidic conditions. Antimicrob. Agents Chemother. 57, 1428-1433 (2013).

6 Poce, G. et al. Improved BM212 MmpL3 inhibitor analogue shows efficacy in acute murine model of tuberculosis infection. PLoS One 8, e56980 (2013).
7 Hillemann, D., Rüsch-Gerdes, S. \& Richter, E. In vitro-selected linezolidresistant Mycobacterium tuberculosis mutants. Antimicrob. Agents Chemother. 52 800-801 (2008).

8 Coban, A. Y., Bilgin, K., Tasdelen Fisgin, N., Uzun, M. \& Durupinar, B. Effect of meropenem against multidrug-resistant Mycobacterium tuberculosis. J. Chemother. 20, 395-396 (2008)

9 Ekins, S., Freundlich, J. S., Choi, I., Sarker, M. \& Talcott, C. Computational databases, pathway and cheminformatics tools for tuberculosis drug discovery. Trends Microbiol. 19, 65-74 (2011).

10 Goldman, R. C. Why are membrane targets discovered by phenotypic screens and genome sequencing in Mycobacterium tuberculosis? Tuberculosis 93, 569-588 (2013).

11 Dashti, Y., Grkovic, T. \& Quinn, R. J. Predicting natural product value, an exploration of anti-TB drug space. Nat. Prod. Rep. 31, 990-998 (2014).

12 Makarov, V. et al. Towards a new combination therapy for tuberculosis with next generation benzothiazinones. EMBO Mol. Med. 6, 372-383 (2014).

13 Takacs-Novak, K. in Physico-Chemical Methods in Drug Discovery and Development (ed. Mandic E.) 2-52 International Association of Physical Chemists (IAPC) Publishing, Zagreb, Croatia, (2012).

14 Gillespie, S. H. et al. Four-month moxifloxacin-based regimens for drug-sensitive tuberculosis. N. Engl. J. Med. 371, 1577-1587 (2014).

15 Merle, C. S. et al. A four-month gatifloxacin-containing regimen for treating tuberculosis. N. Engl. J. Med. 371, 1588-1598 (2014).

16 Jindani, A. et al. High-dose rifapentine with moxifloxacin for pulmonary tuberculosis. N. Engl. J. Med. 371, 1599-1608 (2014).

17 World Health Organization. Global Tuberculosis Report 2014. Chapter 9: Research and development. WHO/HTM/TB/2014.08.

18 Williams, K. et al. Sterilizing activities of novel combinations lacking first- and second line drugs in a murine model of tuberculosis. Antimicrob. Agents Chemother. 56, 3114-3120 (2012)

19 Tasneen, R. et al. Contribution of the nitroimidazoles PA-824 and TBA-354 to the activity of novel regimens in murine models of tuberculosis. Antimicrob. Agents Chemother. 59, 129-135 (2015).

20 Sarathy, J., Dartois, V., Dick, T. \& Gengenbacher, M. Reduced drug uptake in phenotypically resistant nutrient-starved nonreplicating Mycobacterium tuberculosis. Antimicrob. Agents Chemother. 57, 1648-1653 (2013).

21 Bacon, J. et al. Non-replicating Mycobacterium tuberculosis elicits a reduced infectivity profile with corresponding modifications to the cell wall and extracellular matrix. PLoS One 9, e87329 (2014).

22 Daniel, J., Sirakova, T. \& Kolattukudy, P. An acyl-CoA synthetase in Mycobacterium tuberculosis involved in triacylglycerol accumulation during dormancy. PLoS One 9, e114877 (2014).

23 Dartois, V. The path of anti-tuberculosis drugs: from blood to lesions to mycobacterial cells. Nat. Rev. Microbiol. 12, 159-167 (2014).

24 Lenaerts, A., Barry, C. E. 3rd \& Dartois, V. Heterogeneity in tuberculosis pathology, microenvironments and therapeutic responses. Immunol. Rev. 264, 288-307 (2015).

25 Lakshminarayana, S. B. et al. Comprehensive physicochemical, pharmacokinetic and activity profiling of anti-TB agents. J. Antimicrob. Chemother. 70, 857-867 (2015).

Supplementary Information accompanies the paper on The Journal of Antibiotics website (http://www.nature.com/ja) 